\title{
Sobrecarga e sintomatologia depressiva em familiares cuidadores de pessoas dependentes de álcool e outras drogas
}

\section{Burden and depressive symptomatology in family caregivers of people dependent on alcohol and other drugs}

\author{
Fernanda Borges Bessa (orcid.org/0000-0001-9815-7513) ${ }^{1}$ \\ Marina Bandeira (orcid.org/0000-0002-7778-6078)2 \\ Tatiana Cury Pollo (orcid.org/0000-0002-8673-2821)3 \\ Daniela Carine Ramires de Oliveira (orcid.org/0000-0002-9573-8424) 4
}

\begin{abstract}
Resumo
Este estudo avaliou a sobrecarga subjetiva e a sintomatologia depressiva de familiares cuidadores de pessoas com dependência de álcool e outras drogas. Foram entrevistados 50 familiares de pacientes de uma instituição filantrópica de tratamento para abuso de substâncias psicoativas. Utilizou-se a escala de avaliação da sobrecarga Burden Interview, o Inventário de Depressão de Beck e um questionário sociodemográfico e clínico. A maioria dos familiares apresentou sobrecarga entre leve a moderada e grave, e aproximadamente metade apresentou sintomatologia depressiva entre leve e grave. Os preditores da sintomatologia depressiva, identificados pela análise de regressão linear múltipla, foram: em primeiro lugar, o grau de sobrecarga e, em seguida, as variáveis: baixa renda, não viver com um companheiro, avaliação negativa do relacionamento com o paciente e maior número de comportamentos problemáticos do paciente. Esses resultados indicam a necessidade de os serviços desenvolverem intervenções psicossociais que diminuam o grau de sobrecarga dos familiares cuidadores e melhorem o relacionamento com os pacientes, de forma a prevenir o desenvolvimento de uma sintomatologia depressiva.
\end{abstract}

Palavras-chave: Cuidadores familiares. Sobrecarga. Depressão. Dependência de substâncias psicoativas. Avaliação de serviços.

\footnotetext{
Abstract

This study evaluated the subjective burden and depressive symptomatology in family caregivers of patients with alcohol and other drugs dependence. Fifty family members of patients from a philanthropic institution for the

${ }^{1}$ Universidade Federal de São João del-Rei, São João del-Rei, Brasil. E-mail: fernandabbessa@ gmail.com.

${ }^{2}$ Universidade Federal de São João del-Rei, São João del-Rei, Brasil. E-mail: bandeira@ufsj.edu.br.

${ }^{3}$ Universidade Federal de São João del-Rei, São João del-Rei, Brasil. E-mail: tpollo@ufsj.edu.br.

${ }^{4}$ Universidade Federal de São João del-Rei, São João del-Rei, Brasil. E-mail: ramires.daniela@ gmail.com.
} 
treatment of substance abuse were interviewed. The Burden Interview for overload assessment scale, the Beck Depression Inventory and a sociodemographic and clinical questionnaire were used. Most family members had a burden between mild to moderate and severe, and approximately half had depressive symptoms between mild and severe. The predictors of depressive symptomatology identified by multiple linear regression analysis, were: first, the degree of burden and, then, the variables: low income, not living with a partner, negative assessment of the relationship with the patient and greater number of problematic patient behaviors. These results indicate the need for services to develop psychosocial interventions that reduce the degree of burden on family caregivers and improve the relationship with patients, in order to prevent the development of depressive symptoms.

Keywords: Family caregivers. Burden. Depression. Dependence on psychoactive substances. Services evaluation. 


\section{Introdução}

Os transtornos mentais e os decorrentes do uso de álcool e outras drogas apresentam alta prevalência e estão associados à morte prematura e a anos vividos com incapacidade, impactando nas famílias e na comunidade (World Health Organization \& Calouste Gulbenkian Foundation, 2017). Estima-se que, para cada usuário de álcool ou outras drogas, quatro a cinco pessoas sejam afetadas direta ou indiretamente, incluindo cônjuges, companheiros, filhos e pais (Soccol et al., 2013). A presença de uma pessoa dependente na família acarreta diversas modificações na vida familiar, seja nas relações interpessoais, vida profissional, atividades de lazer ou no aspecto financeiro (Mattoo, Nebhinani, Anil Kumar, Basu, \& Kulhara, 2013), desencadeando elevados níveis de estresse. Os familiares precisam lidar com a instabilidade comportamental do usuário, gerando sobrecarga, sentimentos de exaustão, insegurança e impotência (Soccol et al., 2014; Oliveira \& Mendonça, 2012).

O cuidado que os familiares provêm é multifacetado e inclui assistência financeira, cuidado com alimentação e vestuário, higiene corporal, administração da medicação, monitoramento dos comportamentos problemáticos e prevenção de acidentes domiciliares (Mannelli, 2013; Soccol et al., 2013). Frequentemente, a responsabilidade pelos cuidados recai sobre um dos membros da família, no papel de cuidador, resultando em sobrecarga (Marcon, Rubira, Espinosa, Belasco \& Barbosa, 2012). Essa situação envolve eventos estressantes por tempo prolongado, podendo contribuir para o desenvolvimento da depressão (Maciel, Melo, Dias, Silva \& Gouveia, 2014).

A sobrecarga familiar refere-se às consequências resultantes do papel de cuidador e é composta por duas dimensões. A dimensão objetiva envolve consequências observáveis, como perdas financeiras, perturbações na rotina diária e excesso de tarefas de cuidado. A dimensão subjetiva envolve a percepção do familiar de que o papel de cuidador constitui uma fonte de desconforto, preocupação ou tensão psicológica (Barroso, 2014; Tessler \& Gamache, 2000).

A depressão também pode resultar do papel de cuidador que o indivíduo desempenha. No estudo de Biegel, Katz-Saltzman, Meeks, Brown e Tracy (2010), familiares de dependentes de álcool e outras drogas apresentaram níveis de sintomatologia depressiva duas vezes maiores do que a população em geral. No entanto, essa variável tem sido mais estudada em cuidadores de pacientes psiquiátricos ou com Alzheimer e demência (Biegel et al., 2010). A depressão do cuidador pode prejudicar, também, os cuidados prestados ao paciente (Maciel et al., 2014).

A sobrecarga e a sintomatologia depressiva constituem as duas principais variáveis aferidas em pesquisas que investigaram o impacto do papel de cuidador, e alguns estudos têm avaliado a relação entre elas (Clyburn, Stones, Hadjistavropoulos \& Tuokko, 2000; Sherwood, Given, Given \& Eye, 2005). Os resultados mostram uma relação significativa entre a sobrecarga e a sintomatologia depressiva, para cuidadores de pacientes com transtornos 
neurológicos (Cotelo, Rodríguez, Pérez, Iglesias \& Lago, 2015; Santos et al., 2014; Sirari, Dhar, Vishwakarma \& Tripathi, 2014), transtornos psiquiátricos (Dias, 2011; Thunyadee, Sitthimongkol, Sangon, Chai-Aroon \& Hegadoren, 2015) e dependência de substâncias psicoativas (Li et al., 2013).

Clyburn et al. (2000) desenvolveram e testaram um modelo explicativo sobre a relação entre essas duas variáveis, por meio de técnicas de modelagem de equações estruturais. Esse modelo postula que os eventos estressores da situação de cuidar funcionam como antecedentes para a sobrecarga subjetiva que, por sua vez, contribuem como antecedente para o desenvolvimento da sintomatologia depressiva. Segundo Clyburn et al. (2000), os resultados encontrados dão suporte a esse modelo teórico, ao passo que o modelo inverso, no qual a sintomatologia depressiva mediaria a sobrecarga, não foi corroborado pelos resultados. Estudos posteriores, como os de Sherwood et al. (2005) e O'Rourke e Tuokko (2004), encontraram resultados semelhantes. No estudo de Sherwood et al. (2005), também baseado em modelagem de equações estruturais, à medida que o grau de sobrecarga do familiar cuidador aumentava, o nível de sintomatologia depressiva também aumentava, e a direção inversa não foi corroborada pelos dados. $\mathrm{O}$ estudo longitudinal de O'Rourke e Tuokko (2004) mostrou, também, que a sobrecarga dos familiares era variável preditora de futura sintomatologia depressiva.

A sobrecarga e a sintomatologia depressiva dos familiares são duas variáveis utilizadas na avaliação de serviços de saúde mental, tendo em vista que serviços com tratamentos mais efetivos para os pacientes e atendimento aos familiares resultam em menores níveis dessas variáveis (Bandeira, Lima \& Barroso, 2014; Eloia, Oliveira, Eloia, Lomeo \& Parente, 2014; Thornicroft \& Tansella, 2010). A inclusão das famílias na avaliação dos serviços é uma das 10 recomendações da Organização Mundial de Saúde (OMS) para a saúde mental no mundo (OMS, 2001).

Visando identificar estudos que tenham avaliado essas duas variáveis em familiares cuidadores de pessoas dependentes de álcool e outras drogas, foi realizada, em 2016, uma busca nos indexadores SciElo, Lilacs, Pubmed, BVS, Medline, PsycInfo, com os descritores "sobrecarga", "depressão", "sintomas depressivos", "familiares", “cuidadores", "substâncias" e "drogas", bem como os termos correspondentes em inglês. Foram encontrados 12 estudos, dos quais oito avaliaram apenas a sobrecarga dos familiares (Biegel, Ihsler, Katz \& Johnson, 2007; Hoertel \& Limosin, 2014; Lamichhane, Shyangwa \& Shakya, 2008; Malik, Kumar, Sidhu, Sharma \& Gulia, 2012; Mattoo et al., 2013; Nebhinani, Anil, Mattoo \& Basu, 2013; Pereira \& Soares, 2011; Shyangwa, Tripathi \& Lal, 2008) - um estudo avaliou apenas os sintomas depressivos (Maciel et al., 2014) e somente três investigaram as duas variáveis (Biegel et al., 2010; Li et al., 2013; Marcon et al., 2012). Entre esses três estudos, somente as duas pesquisas internacionais (Li et al., 2013; Biegel et al., 2010) investigaram a relação entre essas variáveis. No estudo de Li (2013), a sobrecarga foi a principal preditora da sintomatologia depressiva, mas no estudo de Biegel et al. (2010), 
a sobrecarga não constituiu uma variável preditora significativa. Nenhum estudo nacional investigou essa relação. No entanto, as pesquisas citadas apontam para a importância de identificar o efeito da sobrecarga na saúde mental dos familiares cuidadores, pois resultados dessa investigação podem indicar ações especificas a serem usadas com familiares, visando diminuir seu grau de sobrecarga, com impacto positivo em suas condições de vida, assim como maior disponibilidade de oferecer cuidados aos pacientes.

Tendo em vista a relevância social dessa temática, a carência de pesquisas nessa área e a contradição de resultados encontrados, o presente estudo teve como objetivos: 1. Avaliar o nível de sobrecarga subjetiva em familiares de dependentes de substâncias psicoativas. 2. Avaliar seu nível de sintomatologia depressiva. 3. Investigar a relação entre essas variáveis. 4. Identificar os fatores preditores da sintomatologia depressiva dos familiares.

\section{Método}

O presente estudo consistiu em uma pesquisa avaliativa, do tipo somativa, que avalia os resultados de um serviço, programa ou intervenção, que, nesta pesquisa, consistiu nos graus de sobrecarga e de sintomatologia depressiva apresentados por familiares cuidadores de pacientes de uma instituição filantrópica para tratamento de dependência de álcool e outras drogas. Trata-se de um estudo do tipo correlacional, ou seja, que investiga a relação entre variáveis naturais, sem a manipulação experimental. Foi utilizado, também, o delineamento de comparação com grupo estático, que compara grupos naturais, quanto às características sociodemográficas e clínicas dos sujeitos (Contandriopoulos, Champagne, Pontvin, Denis \& Boyle, 1994).

\section{Participantes}

A amostra foi constituída por familiares cuidadores de pacientes dependentes de álcool e outras drogas atendidos em uma instituição filantrópica de tratamento, em uma cidade de médio porte de Minas Gerais, que atenderam aos critérios de inclusão e exclusão adotados. Os critérios de inclusão foram: a) ser ambos os sexos; b) ter idade igual ou superior a 18 anos; c) estar residindo com o paciente; d) ser indicado pelo serviço como seu principal cuidador, aquele responsável pelos cuidados diários ao paciente; e) terem os pacientes sido diagnosticados para "Transtornos Mentais e Comportamentais devido ao uso de Substâncias Psicoativas Síndrome de Dependência" (F10.2-F19.2), de acordo com a Classificação Estatística Internacional de Doenças e Problemas Relacionados à Saúde - CID-10 (OMS, 1998). Foram selecionados somente os casos de dependência de álcool e/ou substâncias psicoativas ilícitas. Os critérios de exclusão foram: dificuldades em compreender as questões dos instrumentos, não aceitar assinar o Termo de Consentimento Livre e Esclarecido, ser cuidador de paciente que estivesse internado nos últimos 15 dias anteriores à entrevista.

A amostra foi não probabilística, do subtipo acidental, na qual os sujeitos são recrutados segundo sua presença em um 
determinado local e momento (Contandriopoulos et al., 1994). Foi calculada previamente a necessidade de uma amostra de 47 sujeitos para garantir o poder da amostra de 95\%, segundo fórmula de cálculo amostral de Miot (2011) para estudos que investigam a relação entre variáveis quantitativas. Preencheram os critérios de inclusão e exclusão 100 familiares, dos quais nove não puderam ser entrevistados devido a dificuldades de contato por telefone, 18 se recusaram a participar, 20 não tinham disponibilidade para se deslocar para os locais das entrevistas e três familiares não estavam disponíveis durante o período da coleta de dados da pesquisa. Subtraindo-se esses números, a amostra constituiu-se, então, de 50 familiares entrevistados, portanto, acima do número calculado previamente para garantir o poder da amostra. Os familiares não receberam nenhum tipo de incentivo por sua participação. Somente os participantes que tiveram custos com deslocamento para os locais de realização das entrevistas foram reembolsados.

A idade média da amostra de familiares era de 52,22 anos (DP $=15,39)$, variando de 25 a 83 anos. A maioria era do sexo feminino $(84 \%)$, sendo casadas ou vivendo como casadas $(66 \%)$. Os familiares eram, na maioria, pais e mães dos pacientes $(60 \%)$ ou cônjuges $(32 \%)$ e a maioria tinha outros filhos (94\%). Quanto à escolaridade, $\quad 96 \%$ dos respondentes frequentaram a escola, sendo que 56\% tinham, no mínimo, o Ensino Fundamental Completo. Aproximadamente metade $(52 \%)$ não estava trabalhando, mas a maioria tinha renda própria (76\%), com valor mensal médio de $\mathrm{R} \$ 1.618,29$ $(\mathrm{DP}=1.043,47)$. Os familiares cuidavam dos pacientes, em média, há 9,26 anos (DP = 9,01), sendo que 64\% recebiam ajuda para isso.

Os pacientes, sob cuidados dos familiares, tinham idade média de 35,14 anos $(\mathrm{DP}=11,18)$, variando de 15 a 63 anos. $\mathrm{A}$ maioria era do sexo masculino (90\%), solteiros $(52 \%)$ ou eram casados ou vivendo como casados (36\%). Todos frequentaram a escola e 64\% tinham o Ensino Fundamental Completo. Metade dos pacientes (50\%) estava trabalhando, $36 \%$ não tinham renda própria e 52\% recebiam até dois salários mínimos. Os prontuários indicaram que $52 \%$ dos pacientes tinham diagnóstico para dependência de múltiplas substâncias e 30\% para dependência de álcool. As durações médias do transtorno e do tratamento eram de 14,04 anos $(\mathrm{DP}=10,40) \mathrm{e}$ 5,58 anos $(\mathrm{DP}=7,37)$, respectivamente. Mais da metade dos pacientes estava abstinente (62\%). Os pacientes apresentavam, em média, 8,32 (DP $=6,54)$ comportamentos problemáticos.

\section{Instrumentos}

\section{Escala Burden Interview (BI)}

Esta escala, elaborada por Zarit, Reever e Bach-Peterson (1980), é um instrumento de avaliação unifatorial da sobrecarga subjetiva dos cuidadores, validada para o Brasil por Scazufca (2002) e por Taub, Andreoli e Bertolucci (2004) e tem 22 itens que avaliam os seguintes aspectos: saúde, vida social e pessoal, situação financeira, bem-estar emocional e relações interpessoais. As alternativas de resposta avaliam a frequência de ocorrência dos itens, em uma escala tipo Likert de cinco pontos: $0=$ nunca; $1=$ raramente; $2=$ 
algumas vezes; $3=$ frequentemente e $4=$ sempre. $\mathrm{O}$ último item avalia o quanto o familiar se sente sobrecarregado, com alternativas de resposta em uma escala tipo Likert de cinco pontos: $0=$ nem um pouco; $1=$ um pouco; $2=$ moderadamente; $3=$ muito e $4=$ extremamente. $\mathrm{O}$ escore global é obtido pela soma dos escores dos itens, variando de 0 a 88 , com classificações nas seguintes categorias: mínima ou ausente (0-20), leve a moderada (2140), moderada a grave (41-60) e grave (61-88) (Adewuya, Owoeye \& Erinfolami, 2011). Essa escala tem qualidades psicométricas satisfatórias de validade e fidedignidade (Scazufca, 2002; Taub et al., 2004).

\section{Inventário de Depressão de Beck - segunda edição (BDI-II)}

Esta escala foi elaborada por Beck, Steer e Brown (1996) para avaliar a intensidade de sintomas depressivos em pacientes psiquiátricos e na população em geral e foi adaptada e validada para o contexto brasileiro por Gorenstein, Wang, Argimon e Werlang (2014), possuindo 21 itens que avaliam comportamentos e atitudes, distribuídos em duas subescalas ou fatores: Cognitivo-Afetivo e Somato-Afetivo (Gorenstein et al., 2014). As alternativas de resposta são dispostas em uma escala do tipo Likert de quatro pontos, que varia de 0 a 3. O escore global é obtido por meio da soma dos itens e pode variar de 0 a 63, classificados nas seguintes categorias, conforme Gorenstein et al. (2014): mínima ou ausente (013), leve (14-19), moderada (20-28) e grave (2963). A escala apresenta qualidades psicométricas de validade e fidedignidade (Gorenstein et al., 2014).

\section{Questionário sociodemográfico}

Foi utilizado um questionário para avaliar as variáveis sociodemográficas e de condições de vida dos familiares e dos pacientes, tais como idade, sexo, estado civil, renda e escolaridade, entre outros. Foram coletados, também, dados referentes às características clínicas dos pacientes, como a(s) droga(s) de uso, duração da doença, número e duração de internações ou outros tratamentos, entre outros. Esse questionário foi baseado em dados da literatura e testado previamente em um estudo piloto.

\section{Procedimentos de coleta de dados}

A coleta de dados foi realizada pela pesquisadora e duas estagiárias graduandas em Psicologia, previamente treinadas, sendo realizado um estudo piloto com $10 \%$ da amostra para verificar a compreensão do questionário sociodemográfico. O recrutamento dos familiares e o agendamento das entrevistas foram realizados por telefone. A aplicação dos instrumentos de medida foi feita oralmente, sendo as perguntas lidas e as respostas anotadas pelas entrevistadoras, em entrevistas individuais presenciais realizadas no serviço de saúde mental, na residência do familiar ou na instituição onde foi realizada a pesquisa, conforme a disponibilidade dos familiares. Cada entrevista durou, em média, uma hora.

\section{Análise de dados}


As análises estatísticas foram realizadas com o Software Statistical Program for Social Sciences (SPSS) versão 13.0, adotando-se um nível de significância $p<0,05$ (Levin, 1987). O teste Kolmogorov-Smirnov indicou que os escores globais da BDI-II apresentaram distribuição normal (K-S $=0,864, p=0,445$ ), justificando o uso de testes paramétricos. Para a descrição da amostra e dos escores das escalas BI e BDI-II, foram feitas análises estatísticas descritivas, com cálculo de médias, desvios padrão (DP) e porcentagens. O Teste Quiquadrado de Aderência foi usado para comparar as porcentagens de casos nas categorias de sobrecarga e de sintomatologia depressiva, sendo esta comparada entre subgrupos de diferentes graus de sobrecarga, por meio da Análise de Variância (Anova) e teste Post Hoc de Tukey.

Para selecionar as variáveis que seriam incluídas na Regressão Linear Múltipla, foram feitas análises univariadas usando o teste $t$ de Student para amostras independentes e correlação de Pearson. Foram selecionadas as variáveis significativas $(\mathrm{p}<0,05)$ e as não significativas com valores de $p$ entre 0,5 e 0,25 , pois, segundo Hosmer e Lemeshow (2000), estas podem se tornar significativas na análise de regressão. Os modelos de regressão foram construídos com o método Stepwise, sendo a normalidade dos resíduos de cada modelo de regressão analisada pelo teste KolmogorovSmirnov e o índice Durbin-Watson (DW).

\section{Considerações éticas}

A pesquisa foi aprovada pela Comissão de Ética em Pesquisa Envolvendo Seres Humanos (Cepes) da instituição onde foi realizada a pesquisa (CAAE n. 53022116.1.0000.5151) e pela coordenação do serviço de saúde, onde os familiares foram identificados. A coleta de dados ocorreu de acordo com os parâmetros éticos, conforme a Resolução n. 466/12 do Conselho Nacional de Saúde (CNS). A participação dos familiares foi voluntária e a coleta de dados realizada depois da leitura e assinatura do Termo de Consentimento Livre e Esclarecido (TCLE).

\section{Resultados}

\section{Avaliação do grau de sobrecarga subjetiva}

O escore global médio de sobrecarga subjetiva foi de 34,80 (DP $=19,94)$ em uma faixa que pode variar de 0 a 88 . A análise das porcentagens de familiares classificadas segundo as categorias de intensidade de sobrecarga da escala mostrou que 28\% deles apresentaram sobrecarga mínima ou ausente, 36\% sobrecarga leve a moderada, $26 \%$ moderada a grave e $10 \%$ apresentou sobrecarga grave. Portanto, somando as duas últimas categorias, observa-se que aproximadamente um terço dos familiares apresentou sobrecarga de moderada a grave. A análise estatística por itens indicou que a maioria dos familiares apresentou sobrecarga elevada (escores 3 e 4) com relação aos itens que expressavam os seguintes sentimentos: de que o paciente depende dele (64\%); de receio pelo futuro do paciente (48\%); de que o paciente espera que cuide dele como se fosse a única pessoa de quem pode depender (48\%); de não 
ter dinheiro suficiente para cuidar do paciente $(48 \%)$.

\section{Avaliação do nível de sintomatologia depressiva}

O escore global de sintomatologia depressiva dos familiares foi de $18(\mathrm{DP}=12,86)$ numa faixa que pode variar entre 0 a 63 . $O$ escore médio na subescala A (CognitivoAfetivo) foi de 7,38 (DP = 6,29), e na subescala B (Somato-Afetivo) foi de 6,42 (DP $=4,36$ ). Não foi encontrada diferença estatisticamente significativa entre os escores das duas subescalas. Quanto às categorias de classificação da intensidade da sintomatologia depressiva, segundo as categorias estabelecidas pela escala, observou-se que $44 \%$ dos familiares apresentavam sintomatologia depressiva mínima ou ausente, 16\% sintomatologia leve, 18\% sintomatologia moderada e $22 \%$ sintomatologia depressiva grave. Portanto, observa-se que aproximadamente um terço dos familiares apresentou uma sintomatologia depressiva de moderada a grave.

\section{Relação entre sobrecarga e sintomatologia depressiva}

Obteve-se uma correlação positiva significativa entre os escores globais de sobrecarga e de sintomatologia depressiva $(r=$ $0,66 ; p \leq 0,001)$. O escore global de sobrecarga também se correlacionou significativamente com os escores das duas subescalas de sintomatologia depressiva: Cognitivo-Afetivo $(r$ $=0,64 ; p \leq 0,001)$ e Somato-Afetivo $(r=0,63 ; p$ $\leq 0,001)$.

A Tabela 1 apresenta os resultados dos escores de sintomatologia depressiva observados nos subgrupos que apresentaram diferentes categorias de sobrecarga. Houve também diferença significativa entre eles quanto aos escores globais de sintomatologia depressiva, $F(3,46)=11,57, p \leq 0,001$. 
Tabela 1. Comparação dos escores globais de sintomatologia depressiva entre as categorias de sobrecarga, por meio da Análise de Variância (Anova) a e teste Post Hoc de Tukey

\begin{tabular}{|c|c|c|}
\hline \multirow{2}{*}{ Categorias de sobrecarga subjetiva (BI) } & \multicolumn{2}{|c|}{ Escore Global - BDI-II } \\
\hline & Média (DP) & $p$ \\
\hline Mínima ou ausente & $11,07(8,94)$ & \multirow{2}{*}{0,931} \\
\hline Leve a moderada & $13,22(8,59)$ & \\
\hline Mínima ou ausente & $11,07(8,94)$ & \multirow{2}{*}{$0,005^{* *}$} \\
\hline Moderada a grave & $24,77(13,29)$ & \\
\hline Mínima ou ausente & $11,07(8,94)$ & \multirow{2}{*}{$0,000^{* * *}$} \\
\hline Grave & $37,00(7,18)$ & \\
\hline Leve a moderada & $13,22(8,59)$ & \multirow{2}{*}{$0,014^{*}$} \\
\hline Moderada a grave & $24,77(13,29)$ & \\
\hline Leve a moderada' & $13,22(8,59)$ & \multirow{2}{*}{$0,000 * * *$} \\
\hline Grave & $37,00(7,18)$ & \\
\hline Moderada a grave & $24,77(13,29)$ & \multirow{2}{*}{0,108} \\
\hline Grave & $37,00(7,18)$ & \\
\hline
\end{tabular}

Nota: BDI-II = Inventário de depressão de Beck; BI = Escala Burden Interview. Categorias de sobrecarga subjetiva: Minima ou ausente (0-20); Leve a moderada (21-40); Moderada a grave (41-60); Grave (6188).

a Anova: $F(3,46)=11,57 ; p \leq 0,001$.

$* p \leq 0,05 . * * p \leq 0,01 . * * * p \leq 0,001$.

Fonte: Elaborada pelas autoras.

$\mathrm{Na}$ comparação entre pares de subgrupos (teste Post Hoc de Tukey), o nível de sintomatologia depressiva foi significativamente mais elevado entre os familiares com sobrecarga grave $(p \leq 0,001)$, quando comparados aos de sobrecarga leve a moderada e sobrecarga mínima ou ausente. O nível de sintomatologia depressiva foi, também, significativamente mais elevado para o subgrupo de familiares com sobrecarga moderada a grave, quando comparado aos subgrupos com sobrecarga leve a moderada $(p \leq 0,05)$ e mínima ou ausente $(p \leq$ $0,01)$. Esses resultados indicam, portanto, em duas análises estatísticas distintas, uma relação significativa entre o grau de sobrecarga e o nível de sintomatologia depressiva.

\section{Análises multivariadas: fatores preditores da sintomatologia depressiva}

A Tabela 2 apresenta os resultados da análise de regressão que avaliaram as características preditoras da sintomatologia depressiva dos familiares. O modelo final de regressão indicou que o grau de sobrecarga dos familiares $(\beta=0,48)$ foi o fator preditivo mais importante para explicar o seu nível de sintomatologia depressiva. 
Tabela 2. Análise de Regressão Linear Múltipla do grau de sintomatologia depressiva em relação às características dos familiares

\begin{tabular}{|c|c|c|c|c|c|c|}
\hline Variáveis associadas & Beta (B) & DP & $\operatorname{Beta}(\beta)$ & $t$ & $P$ & \\
\hline Constante & 30,591 & 7,23 & - & 4,23 & $0,000 * * *$ & \multirow{6}{*}{$\begin{array}{l}\mathrm{R}^{2}=0,54 \\
\mathrm{~F}=11,64 \\
p=0,000^{* * *} \\
\mathrm{DW}=1,93\end{array}$} \\
\hline BI - Escore Global & 0,304 & 0,08 & 0,48 & 4,02 & $0,000^{* * *}$ & \\
\hline Avaliação do & & & & & & \\
\hline $\begin{array}{l}\text { relacionamento com o } \\
\text { paciente }\end{array}$ & $-14,512$ & 5,51 & $-0,32$ & $-2,63$ & $0,013^{*}$ & \\
\hline Valor da renda $(\mathrm{R} \$)$ & $-0,004$ & 0,00 & $-0,31$ & $-2,63$ & $0,013^{*}$ & \\
\hline $\begin{array}{l}\text { Vive com } \\
\text { companheiro(a) }\end{array}$ & $-6,160$ & 2,95 & $-0,24$ & $-2,09$ & $0,044^{*}$ & \\
\hline
\end{tabular}

Nota: $\mathrm{BI}=$ Escala Burden Interview.

$* \mathrm{p} \leq 0,05 .{ }^{* * *} \mathrm{p} \leq 0,001$.

Fonte: Elaborada pelas autoras.

As outras variáveis que foram identificadas como preditoras do nível de sintomatologia depressiva, em ordem decrescente de importância, foram: a avaliação negativa do familiar quanto ao relacionamento com o paciente $(\beta=-0,32)$, ter menor valor $\mathrm{da}$ renda $(\beta=-0,31)$ e não viver com um companheiro $(\beta=-0,24)$. Essas variáveis explicaram $54 \%$ da variância dos dados do nível de sintomatologia depressiva.

A Tabela 3 apresenta a análise de regressão da sintomatologia depressiva dos familiares em função das características dos pacientes e do grau de sobrecarga dos familiares. O modelo final de regressão explicou $84 \%$ da variância dos dados. 
Tabela 3. Análise de Regressão Linear Múltipla do grau de sintomatologia depressiva dos familiares em relação à sua sobrecarga subjetiva e às características dos pacientes

\begin{tabular}{lcccccl}
\hline \multicolumn{1}{c}{ Variáveis associadas } & Beta (B) & DP & Beta $(\boldsymbol{\beta})$ & $\boldsymbol{t}$ & $\boldsymbol{p}$ & \\
\hline BI - Escore Global & 0,346 & 0,07 & 0,61 & 4,65 & $0,000^{* * *}$ & $\mathrm{R}^{2}=0,84$ \\
Número de & & & & & & $\mathrm{F}=85,69$ \\
$\begin{array}{l}\text { comportamentos } \\
\text { problemáticos atuais }\end{array}$ & 0,867 & 0,33 & 0,34 & 2,61 & $0,014^{*}$ & $p=0,000^{* * *}$ \\
& & & & & & $\mathrm{DW}=1,93$ \\
\hline
\end{tabular}

Nota: BI = Escala Burden Interview.

$* p \leq 0,05 . * * * p \leq 0,001$.

Fonte: Elaborada pelas autoras

Nesse modelo de análise das características dos pacientes, o grau de sobrecarga dos familiares $(\beta=0,61)$ constituiu novamente o fator preditivo mais importante para explicar o seu nível de sintomatologia depressiva, seguido pela variável maior número de comportamentos problemáticos atuais dos pacientes $(\beta=0,34)$.

\section{Discussão}

No presente estudo, os familiares apresentaram uma média global de sobrecarga leve a moderada e dados de porcentagens indicando que $36 \%$ apresentaram sobrecarga entre moderada e grave, segundo as categorias de classificação da escala BI, apresentadas por Adewuya et al. (2011). Hoertel e Limosin (2014) encontraram resultados semelhantes, com o mesmo tipo de população-alvo e mesmo instrumento de medida, tendo obtido uma média de sobrecarga leve a moderada e dados de porcentagens indicando que $22 \%$ dos familiares apresentaram sobrecarga de moderada a grave. No caso das pesquisas feitas com a mesma população-alvo e instrumentos de medida diferentes, algumas obtiveram um nível de sobrecarga semelhante ao do presente estudo (Lamichanne et al., 2008; Biegel et al., 2007, 2010; Malik et al., 2012; Marcon et al., 2012), enquanto outras encontraram um nível de sobrecarga mais elevado (Mattoo et al., 2013; Nebhinani et al., 2013; Shyangwa et al., 2008; Li et al., 2013). A sobrecarga mais elevada dessas últimas pesquisas talvez se explique pelo tipo de amostra estudada, constituída por cuidadores de pacientes com dependência de drogas opiáceas e/ou que ministravam drogas por via intravenosa, que envolve mais comportamentos problemáticos e de alto risco, como a troca de seringas e a consequente exposição ao HIV (Li et al., 2013; Mattoo et al., 2013; Nehbinani et al., 2013; Shyangwa et al., 2008).

O grau de sobrecarga do presente estudo assemelhou-se aos resultados de algumas pesquisas que utilizaram o mesmo instrumento de medida, porém com populações-alvo distintas, tais como cuidadores de pacientes com dependência funcional (Guedes \& Pereira, 2013), demência (Santos et al., 2014), epilepsia (Sirari et 
al., 2014) e insuficiência cardíaca (Zincir et al., 2014). Por outro lado, a sobrecarga observada, no presente trabalho, foi mais elevada do que a encontrada com familiares cuidadores de pacientes com insuficiência cardíaca (Hooley, Butler \& Howlett, 2005; Chung, Pressler, Dunbar, Lennie \& Moser, 2010), e com portadores de deficiência cognitiva leve (Springate \& Tremont, 2011).

Os resultados da escala BDI-II indicaram que $56 \%$ dos familiares apresentavam algum grau de sintomatologia depressiva e 40\% apresentavam níveis de sintomatologia de moderada a grave. Esses resultados são semelhantes aos encontrados nos estudos de Maciel et al. (2014), Marcon et al. (2012) e Biegel et al. (2010), realizados com a mesma populaçãoalvo e instrumentos de medida diferentes. Por outro lado, níveis mais elevados de sintomatologia depressiva foram observados em pesquisas realizadas com amostras de cuidadores de pacientes dependentes de drogas injetáveis, cujos comportamentos de risco podem ser mais estressantes para os familiares ( $\mathrm{Li}$ et al., 2013; Mattoo et al., 2013; Nehbinani et al., 2013; Shyangwa et al., 2008).

Os resultados da sintomatologia depressiva obtidos na presente pesquisa estão em consonância com os encontrados em alguns estudos realizados com o mesmo instrumento de medida, porém, avaliando populações-alvo distintas, tais como familiares de pacientes com Alzheimer (Cotelo et al., 2015) e epilepsia (Sirari et al., 2014). Por outro lado, os resultados do presente estudo foram superiores aos encontrados em pesquisas com populações-alvo de cuidadores de pacientes com insuficiência cardíaca (Chung et al., 2010; Zincir et al., 2014). Esses resultados, assim como os dados da sobrecarga citados anteriormente, indicam que cuidar de um paciente com dependência de álcool e outras drogas pode representar uma tarefa mais desgastante para os familiares, devido às particularidades do transtorno (Maciel et al., 2014).

Os resultados obtidos sobre a relação entre sobrecarga e sintomatologia depressiva confirmaram a hipótese do presente trabalho, em consonância com o modelo teórico de Clyburn et al. (2000). A sobrecarga constituiu a variável preditora mais importante do nível de sintomatologia depressiva dos familiares, comparativamente a outras variáveis. Esses resultados corroboram dados de outros estudos (Clyburn et al., 2000; Dias, 2011; O’Rourke \& Tuokko, 2004; Sherwood et al., 2005; Thunyadee, 2015; Zincir et al., 2014) e demonstram que, para a população-alvo do presente trabalho, o atendimento dos familiares cuidadores deve priorizar intervenções visando à diminuição de sua sobrecarga.

As demais variáveis associadas à sintomatologia depressiva, no presente estudo, foram: avaliação negativa do relacionamento com o paciente, menor valor da renda, não viver com um companheiro e maior número de comportamentos problemáticos dos pacientes. O papel de cuidador pode ser menos estressante quando há um bom relacionamento com o paciente (Clyburn et al., 2000). Quanto à renda, o estresse financeiro constitui um dos fatores de risco que afeta a saúde mental dos familiares (Pinquart \& Sörensen, 2003), principalmente no caso de dependentes de álcool e outras drogas, 
que apresentam frequentemente dificuldades em administrar sua vida financeira (Shyangwa et al., 2008). A variável "não viver com um companheiro" pode significar que os familiares sofrem com menor suporte social, que resulta em níveis mais elevados de sintomatologia depressiva (Clyburn et al., 2000). Quanto aos comportamentos problemáticos dos pacientes, essa variável é fortemente preditora da sintomatologia depressiva de familiares cuidadores de diferentes tipos de pacientes (Clyburn et al., 2000; Pinquart \& Sörensen, 2003). As variáveis referentes a dificuldades de relacionamento com o paciente, ter que lidar com os seus comportamentos problemáticos, assim como falta de suporte social, têm sido identificadas como fatores associados a um maior grau de sobrecarga dos cuidadores (Barroso, 2014), o que sugere que a sobrecarga poderia mediar os efeitos dessas variáveis sobre o grau de sintomatologia depressiva, portanto, fornecendo mais suporte ao modelo teórico de Clyburn et al. (2000), adotado no presente trabalho.

O presente estudo tem algumas limitações, como o fato de se tratar de uma pesquisa do tipo correlacional, que não estabelece relações causais entre variáveis. No entanto, ela permite avaliar relações preditivas, de como os níveis de uma variável independente podem predizer a variação nos níveis da variável dependente, analisando um conjunto de variáveis simultaneamente e determinando a ordem de importância relativa entre elas (Sherwood et al., 2005). Outra limitação se refere à dificuldade de generalização dos resultados, devido ao uso de uma amostra não aleatória de sujeitos e ao fato de a avaliação ter sido realizada em um serviço que difere dos serviços públicos existentes na rede de saúde. Estudos futuros devem ser desenvolvidos em outros tipos de serviços, em particular os Centros de Atenção Psicossocial - Álcool e Outras Drogas (Caps $\mathrm{AD})$.

\section{Considerações finais}

Pode-se concluir que os familiares cuidadores de pessoas com dependência de álcool e outras drogas apresentaram sobrecarga e sintomatologia depressiva, de intensidade moderada a grave, em aproximadamente um terço da amostra e que a sobrecarga constituiu a principal variável preditora da sintomatologia depressiva. Os dados do presente trabalho contribuem para o aumento do conhecimento da área, tendo em vista a carência de pesquisas anteriores sobre a relação entre a sobrecarga e a sintomatologia depressiva dessa população-alvo.

Os resultados obtidos são importantes, também, porque apontam para a necessidade de os serviços de atenção aos usuários de álcool e outras drogas incluírem, em seu tratamento, ações psicossociais direcionadas aos familiares que envolvam: fornecer informações sobre o transtorno, orientações sobre como lidar com os comportamentos problemáticos do paciente e intervenções para melhorar o relacionamento entre o familiar e o paciente. Essas ações poderiam contribuir para diminuir a sobrecarga dos cuidadores, de forma a reduzir o risco de desenvolvimento de uma sintomatologia depressiva. Esses efeitos contribuiriam para promover melhores condições de vida e saúde 
mental dos familiares cuidadores, resultando em sua maior disponibilidade para desempenhar o papel de cuidadores dos pacientes.

\section{Referências}

Adewuya, A. O., Owoeye, O. A., \& Erinfolami, A. R. (2011). Psychopathology and subjective burden amongst primary caregivers of people with mental illness in SouthWestern Nigeria. Social Psychiatry and Psychiatric Epidemiology, 46, 1251-1256. Doi: 10.1007/s00127-010-0293-0.

Barroso, S. (2014). Sobrecarga de familiares cuidadores de pacientes psiquiátricos. In M. Bandeira, L. Lima \& S. Barroso, Avaliação de serviços de saúde mental: princípios metodológicos, indicadores de qualidade $e$ instrumentos de medida (pp. 187-216). Petrópolis: Editora Vozes.

Bandeira, M., Lima, L. A., \& Barroso, S. M. (2014). Avaliação de serviços de saúde mental: princípios metodológicos, indicadores de qualidade e instrumentos de medida. Petrópolis: Editora Vozes.

Beck, A. T., Steer, R. A., \& Brown, G. K. (1996). Manual for the Beck. Depression Inventory-II. San Antonio, TX: Psychological Corporation.

Biegel, D. E., Ishler, K. J., Katz, S., \& Johnson, P. (2007). Predictors of burden of family caregivers of women with substance use disorders or co-occurring substance and mental disorders. Journal of Social Work Practice in the Addictions, 7, 25-49. Doi:
10.1300/J160v07n01_03.

Biegel, D. E., Katz-Saltzman, S., Meeks, D., Brown, S., \& Tracy, E. M. (2010). Predictors of depressive symptomatology in family caregivers of women with substance use disorders or co-occurring substance use and mental disorders. Journal of Family Social Work, 13(2), 25-44. Doi: 10.1080/10522150903437458.

Clyburn, L. D., Stones, M. J., Hadjistavropoulos, T., \& Tuokko, H. (2000). Predicting caregiver burden and depression in Alzheimer's disease. Journal of Gerontology: Social Sciences, 55B(1), 2-13. Doi: 10.1093/geronb/55.1.S2.

Contandriopoulos, A. P., Champagne, F., Potvin, L., Denis, J. O., \& Boyle, P. (1994). Saber preparar uma pesquisa. São Paulo: Hucitec Abrasco.

Cotelo, N. V., Rodríguez, F. A., Pérez, J. A. F., Iglesias, J. C. A., \& Lago, M. R. (2015). Burden and associated pathologies in family caregivers of Alzheimer's disease patients in Spain. Pharmacy Practice, 13(2), 1-6.

Doi: 10.18549/PharmPract.2015.02.521.

Chung, M. L., Pressler, S. J., Dunbar, S. B., Lennie, T. A., \& Moser, D. K. (2010). Predictors of depressive symptoms in caregivers of patients with heart failure. Journal of Cardiovascular Nursing, 25(5), 411419.

Doi:10.1097/JCN.0b013e3181d2a58d.

Dias, E. A. (2011). Sobrecarga vivenciada por 
familiares cuidadores de pacientes esquizofrenicos e sua relação com a depressão. Dissertação de mestrado, Universidade Nova de Lisboa, Lisboa, Portugal. Recuperado em 24 fevereiro 2017, de https://run.unl.pt/handle/10362/5932.

Eloia, S. C., Oliveira, E. N., Eloia, S. M. C., Lomeo, R. C., \& Parente, J. R. F. (2014). Sobrecarga do cuidador familiar de pessoas com transtorno mental: uma revisão integrativa. Saúde Debate, 38(103), 996-1007. Doi: 10.5935/01031104.20140085.

Gorenstein, C., Wang, Y. P., Argimon, I. L., \& Werlang, B. S. G. (2014). Manual do Inventário de Depressão de Beck - BDIII. São Paulo: Editora Casa do Psicólogo.

Guedes, A. C., \& Pereira, M. G. (2013). Sobrecarga, enfrentamento, sintomas físicos e morbidade psicológica em cuidadores de familiares dependentes funcionais. Revista Latino-Americana de Enfermagem, 21(4), 1-6. Doi: 10.1590/S0104-11692013000400015.

Hoertel, N., \& Limosin, F. (2014). Excessive alcohol consumption: what is the burden on natural caregivers? Encéphale, 40, 1-10. Doi: 10.1016/j.encep.2014.02.007.

Hooley, P. J. D., Butler, G., \& Howlett, J. G. (2005). The relationship of quality of life, depression, and caregiver burden in outpatients with congestive heart failure. Congestive Heart Failure, 11(6), 303-310. Doi 10.1111/j.1527-5299.2005.03620.x.
Hosmer, D. W., \& Lemeshow, S. (2000). Applied logistic regression. New York: John Wiley and Sons.

Lamichhane, N., Shyangwa P. M., \& Shakya R. (2008). Family burden in substance dependence syndrome. Journal of Gandaki Medical College, 1(1), 51. Retrieved February 24, 2017, from https://www.researchgate.net/publicatio n/228333401_Family_burden_in_substan ce_dependence_syndrome.

Levin, J. (1987). Estatística Aplicada às ciências bumanas. São Paulo: Harbra.

Li, L., Tuan, N. A., Liang, L., Lin, C., Farmer, S. C., \& Flore, M. (2013). Mental health and family relations: correlated reports from people who inject drugs and their family members in Vietnam. International Journal of Drug Policy, 24(6), 1-11. Doi: 10.1016/j.drugpo.2013.06.007.

Maciel, S. C., Melo, J. R. F., Dias, C. C. V., Silva, G. L. S., \& Gouveia, Y. B. (2014). Sintomas depressivos em familiares de dependentes químicos. Revista Psicologia: Teoria e Prática, 16(2), 18-28. Recuperado em 24 fevereiro, 2017, de http://pepsic.bvsalud.org/scielo.php?scri $\mathrm{pt}=$ sci_arttext\&pid $=$ S151636872014000200002.

Malik, P., Kumar, N., Sidhu, B. S., Sharma, K. C., \& Gulia, A. D. (2012). Impact of substance dependence on primary caretaker in rural Punjab. Delhi Psychiatry Journal, 15(1), 72-78. Retrieved February 24, 2017, 
from

http://medind.nic.in/daa/t12/i1/daat12i 1p72.pdf.

Mannelli, P. (2013). The burden of caring: drug users \& their families. Indian Journal of Medical Research, 137, 636-638. Retrieved February 24, 2017, from https://www.ncbi.nlm.nih.gov/pmc/artic les/PMC3724243/.

Marcon, S. R., Rubira, E. A., Espinosa, M. M., Belasco, A., \& Barbosa, D. A. (2012). Qualidade de vida e sobrecarga de cuidados em cuidadores de dependentes químicos. Acta Paulista de Enfermagem, 25(2), 7-12. Doi: 10.1590/S010321002012000900002.

Mattoo, S. K., Nebhinani, N., Anil Kumar B. N., Basu, D., \& Kulhara, P. (2013). Family burden with substance dependence: a study from India. Indian Journal Medical Research, 137, 704-711. Retrieved February 24, 2017, from https://www.ncbi.nlm.nih.gov/pmc/artic les/PMC3724250/.

Miot, H. A. (2011). Tamanho da amostra em estudos clínicos e experimentais. Jornal Vascular Brasileiro, 10(4), 275-278. Doi: 10.1590/S1677-54492011000400001.

Nebhinani, N., Anil, B. N., Mattoo, S. K., \& Basu D. (2013). Family burden in injecting versus noninjecting opioid users. Industrial Psychiatry Journal, 22(2), 138-42. Doi: 10.4103/0972-6748.132928.

O’Rourke, N., \& Tuokko, H. A. (2004).
Caregiver burden and depressive symptomatology: the association between constructs over time. Clinical Gerontologist, 27(4), 41-52. Doi: 10.1300/J018v27n04_05.

Oliveira, E. B., \& Mendonça, J. L. S. (2012). Familiar com dependência química e consequente sobrecarga sofrida pela família: pesquisa descritiva. Online Brazilian Journal of Nursing, 11(1). Recuperado em 16 julho, 2014, de http://www.objnursing.uff.br/index.php/ nursing/article/view/3480/html_4.

Organização Mundial de Saúde. (1998). CID-10: Classificação Internacional de Doenças e Problemas Relacionados à Saúde. São Paulo: Edusp.

Organização Mundial de Saúde. (2001). Organização Pan-Americana de Saúde (Opas) - Saúde Mental: Nova Concepção, Nova Esperança. Relatório sobre a Saúde no Mundo. Genebra: Opas/OMS.

Pereira, M. G., \& Soares, A. J. (2011). Sobrecarga em cuidadores informais de dependentes de substâncias: adaptação do Caregiver Reaction Assessment. Psicologia, saúde \& doenças, 12(2), 304-328. Recuperado em 24 fevereiro, 2017, de http://www.scielo.mec.pt/scielo.php?scri $\mathrm{pt}=$ sci_arttext\&pid $=\mathrm{S} 1645$ 00862011000200011.

Pinquart, M., \& Sörensen, S. (2003). Associations of stressors and uplifts of caregiving with caregiver burden and 
depressive mood: a meta-Analysis. Journal of Gerontology: Psychological Sciences, 58B(2), 112-128. Doi: 10.1093/geronb/58.2.P112. Santos, R. L., Sousa, M. F. B., Simões-Neto, J. P., Nogueira, M. L., Belfort, T. T., Torres, B., Rosa, R. D. L., Laks, J., \& Dourado, M. C. N. (2014). Caregivers' quality of life in mild and moderate dementia. Arquivos de Neuro-Psiquiatria, 72(12), 931-937. Doi: 10.1590/0004-282X20140155.

Scazufca, M. (2002). Brazilian version of the Burden Interview scale for the assessment of burden of care in carers of people with mental illnesses. Revista Brasileira de Psiquiatria, 24(1), 12-7. Doi: 10.1590/S1516-44462002000100006.

Sherwood, P. R., Given, C. W., Given, B. A., \& Eye, A. V. (2005). Caregiver burden and depressive symptoms: analysis of common outcomes in caregivers of elderly patients. Journal of Aging and Health, 17(2), 125-147. Doi: $10.1177 / 0898264304274179$.

Shyangwa, P. M., Tripathi, B. M., \& Lal, R. (2008). Family burden in opioid dependence syndrome in tertiary care centre. Journal of Nepal Medical Association, 47(171), 113-119. Doi: 10.4103/09726748.13292.

Sirari, S., Dhar, D., Vishwakarma, R., \& Tripathi, S. (2014). Association between quality of life, depression and caregiver burden in epileptic patients in Haldwani, Uttarakhand, India. International Jounal of
Current Science, 10, 54-60. Recuperado em 24 fevereiro, 2017, de http://www.currentsciencejournal.info/is suespdf/Shiva\%20sirari9.pdf.

Soccol, K. L. S., Terra, M. G., Girardon-Perlini, N. M. O., Ribeiro, D. B., Silva, C. T., \& Camillo, L. A. (2013). O cuidado familial ao indivíduo dependente de álcool e outras drogas. Revista Rene, 14(3), 549-57. Doi: 10.15253/rev\%20rene.v14i3.3431.

Soccol, K. L. S., Terra, M. G., Ribeiro, D. B., Teixeira, J. K. S., Siqueira, D. F., \& Mostardeiro, S. C. T. S. (2014). O cotidiano das relações familiares com indivíduo dependente químico. Cogitare Enfermagem, 19(1), 116-22. Doi: 10.5380/ce.v19i1.35967.

Taub, A., Andreoli, S. B., \& Bertolucci, B. H. (2004). Dementia caregiver burden: reliability of the Brazilian version of the Zarit caregiver burden interview. Cadernos de Saúde Pública, 20(2), 372-376. Doi: 10.1590/S0102-311X2004000200004.

Tessler, R. C. \& Gamache, G. M. (2000). Family experiences with mental illness. Westport: Auburn House.

Thornicroft, G., \& Tansella, M. (2010). Boas práticas em saúde mental. Barueri: Manole.

Thunyadee, C., Sitthimongkol, Y., Sangon, S., Chai-Aroon, T., \& Hegadoren, K. M. (2015). Predictors of depressive symptoms and physical health in caregivers of individuals with schizophrenia. Nursing and Health Sciences, 
17, 412-419. Doi: 10.1111/nhs.12205.

World Health Organization, \& Calouste Gulbenkian Foundation. (2017). Policy options on mental health: A WHOGulbenkian Mental Health platform collaboration. Geneva: World Health Organization.

Zarit, S. H., Reever, K. E., \& Back-Peterson, J. (1980). Relatives of the impaired elderly: Correlates of feelings of burden. The Gerontologist, 20, 649-655. Doi: https://doi.org/10.1093/geront/20.6.649.

Zincir, S. B., Sunbul, M., Zincir, S., Sunbul, E., Oguz, M., Cengiz, F. F., Durmus, E., Kivrak, T., \& Sari, I. (2014). Burden and depressive symptoms associated with adult-child caregiving for individuals with heart failure. Scientific World Journal, 1-6. Doi: 10.1155/2014/641817.

Recebido em: 28/11/2017 Aprovado em: 3/4/2018 